\title{
The IPP RF Source: A High Power, Low Pressure Negative Ion Source For The Neutral Beam Injection System Of ITER
}

\author{
P. Franzen, U. Fantz, W. Kraus, M. Berger, S. Christ-Koch, M. Fröschle, \\ R. Gutser, B. Heinemann, C. Martens, F. Maisberger, P. McNeely, R. Riedl, \\ E. Speth, D. Wünderlich, T. Zacharias
}

Max-Planck-Institut für Plasmaphysik, EURATOM Association, PO Box 1533, D-85740 Garching, Germany

\begin{abstract}
IPP Garching has successfully developed a RF driven negative ion source for the ITER neutral beam injection system. The RF source was chosen recently as the reference source for ITER due to its in principle maintenance-free operation. Current densities of $330 \mathrm{~A} / \mathrm{m}^{2}$ and $230 \mathrm{~A} / \mathrm{m}^{2}$ have been achieved for hydrogen and deuterium, respectively, at a pressure of $0.3 \mathrm{~Pa}$ and an electron/ion ratio of less than 1 for a small extraction area $\left(7.0 \times 10^{-3} \mathrm{~m}^{2}\right)$ and short pulses $(<4 \mathrm{~s})$. The development concentrates now on extending the pulse length and extending the size of the source on two dedicated test facilities. The pulse length can be extended up to one hour at the long pulse test facility having an extraction area of $0.02 \mathrm{~m}^{2}$. The large source test facility is equipped a large RF source with the width and half the height of the ITER beam source in order to demonstrate the homogeneity of a large RF plasma. The paper will give a short overview on the results achieved at the three test facilities of IPP; the underlying physical mechanisms are discussed with an emphasis on electron suppression.
\end{abstract}

Keywords: Neutral Beam Injection, ITER, negative ion source, RF source

PACS: 28.52.Cx, 52.27.Cm, 52.50.Dg, 52.50.Gj

\section{INTRODUCTION}

The neutral beam injection system (NBI) of ITER [1] consists of two heating beams (HNB) and one diagnostic beam (DNB). In order to achieve the required parameters (see table 1), large ion sources delivering several tens Amperes of negative ion current are necessary. In contrast to positive hydrogen ions, negative hydrogen ions have a high neutralization efficiency (60\%) for energies above $200 \mathrm{keV}$ due to the low binding energy of the additional electron $(0.75 \mathrm{eV})$. This low binding energy, however, makes on the other hand the production of negative ions very difficult; the current densities are typically a factor of ten lower than for positive ions. Hence, negative ion sources have to be much larger in size for the same power.

The fundamental processes which lead to negative hydrogen ion production are volume production (dissociative attachment) and surface production, i.e. conversion of $\mathrm{H}$ atoms and positive ions to negative ions on a surface with sufficient low work function. The latter process is being used in all present negative ion based NBI systems because of its much better performance. This is especially the case for the low pressure operation required for negative ion based NBI systems: the stripping losses, i.e. the loss of the additional electron, in the accelerator system due to collisions with the background gas, depend on the source pressure. These stripping losses lead to non-fully accelerated ions and to additional heat loads of the grid system. As a compromise between these stripping losses and source operation, ITER requires a source pressure of $0.3 \mathrm{~Pa}$.

The development of large negative ion sources was started in the early 90's in Japan with filamented arc sources as the basis for the design of the ITER NBI system. Filamented sources, however, suffer from regular maintenance periods (twice per year in ITER) due to the limited lifetime of the filaments. Due to the advantages of the RF source - it is in principle maintenance-free - and due to the good experience with the positive ion based RF sources [2] at the NBI system for ASDEX Upgrade and W7-AS, IPP Garching started the development of a RF driven negative ion source end of the 90's, from 2002 on within a framework of an official EFDA contract. The development was very successful [3,4]: recently, in July 2007, the RF source was chosen by the ITER board as the reference source [5]. 


\section{STATUS OF THE DEVELOPMENT}

The development of the IPP negative hydrogen ion RF source is done at three test facilities in parallel: Current densities of $330 \mathrm{~A} / \mathrm{m}^{2}$ with $\mathrm{H}^{-}$and $230 \mathrm{~A} / \mathrm{m}^{2}$ with $\mathrm{D}^{-}$have been achieved with the IPP RF source on the small test facility BATMAN (Bavarian Test Machine for Negative Ions) at the required source pressure $(0.3 \mathrm{~Pa})$ and electron/ion ratios (see figure 1), but with a small extraction area $\left(70 \mathrm{~cm}^{2}\right)$ and limited pulse length $(<4 \mathrm{~s})$ [3]. The development concentrates now on the extension of source size and extraction area as well as on the extension of the pulse length. This is done at the two other test facilities MANITU (Multi Ampere Negative Ion Test Unit) where the extraction area is about $200 \mathrm{~cm}^{2}$ and the pulse length up to $3600 \mathrm{~s}$, using the same source as it is used at BATMAN, and at the dedicated plasma source test facility RADI, which is equipped with a "half-size" source having the full width and half the height of the ITER source. RADI has no extraction and is devoted to demonstrate the modular concept of the RF source and the plasma uniformity of a large RF source [6]. MANITU was upgraded to cw operation by an active cooling of all parts of the source and has achieved now stable one hour pulses; the parameters however, are still below the ITER requirements [7].

The IPP RF source consists of three parts: the driver, where the RF is coupled to the plasma, the expansion region, where the plasma expands into the actual source body, and the extraction region. The latter two are separated by a magnetic field parallel to the plasma grid, the filter field (see below). The driver is mounted on the back of the source body and consists of a $245 \mathrm{~mm}$ id alumina cylinder with a water-cooled RF coil connected to a $150 \mathrm{~kW}$, $1 \mathrm{MHz}$ oscillator. An internal copper Faraday screen protects the alumina cylinder from the plasma. In order to achieve the required low work function, $\mathrm{Cs}$ is evaporated into the source. Due to the short survival lengths of a few $\mathrm{cm}$ only, only negative $\mathrm{H}$ ions that are produced at the plasma grid can be extracted. The main challenge of negative $\mathrm{H}$ sources for ITER is to create Cs conditions with temporal (long pulse) and spatial (large source) stability. This Cs conditioning is usually done in BATMAN by a slow evaporation rate $(\sim 10 \mathrm{mg} / \mathrm{h})$ for several days; for long pulses at MANITU, however, the Cs dynamics cannot be controlled sufficiently up to now.

\section{NEGATIVE ION PRODUCTION AND ELECTRON SUPPRESSION}

One special physical aspect in negative ion sources is the extraction of electrons together with negative ions. To keep this co-extracted electron current low is an important task, otherwise the accelerated fast electrons would damage the electrodes and would cause neutralization losses. The electrons are filtered out of the beam by magnets in the grid system. The co-extracted electron current and hence the power load on the grids limits the maximum negative ion current for large NBI ion sources. This is especially the case for D operation. Here the electron/ion ratio is larger than for $\mathrm{H}$ (factor 3-5) which is more one can expect from the higher mass and hence the lower velocity of a $\mathrm{D}$ ion [3].

The physics of the ion production and extraction is rather complicated and up to now only partially understood. A further complexity arises from drifts of the plasma in the filter field and the resulting inhomogeneity of the plasma in front of the plasma grid. This leads to an inhomogeneous beam and poor transmission of the beam. 3DMonte Carlo models including the complex electrical potential distribution and the magnetic field configuration are under way [8].

The ions that are created at the plasma grid are accelerated into the source by the sheath potential and are bent back towards the plasma grid by charge exchange collisions and the magnetic fields [8]. The negative ions can be destroyed by collisions with positive ions, atoms and electrons. The latter process can be minimized by reducing the

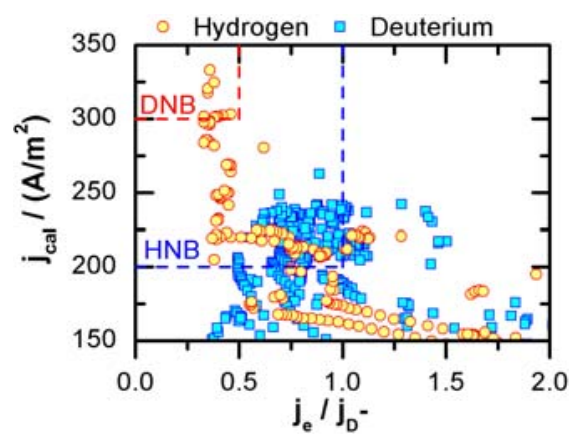

FIGURE 1. Performance of the IPP RF source at BATMAN. Indicated are the ITER requirements for the heating beams (HNB) and for the diagnostic beam (DNB). The internal filter field for $\mathrm{H}$ was lower than for $\mathrm{D}$. 
electron temperature below $2 \mathrm{eV}$, and then atom/ion collisions are the dominant processes. The extraction probability depends then on the total path length of the ion as well as on the local electron/ion/atom temperature and density. The experimental evidence shows that optimum performance can be achieved by a hot plasma grid $\left(\sim 150{ }^{\circ} \mathrm{C}\right)$ and warm source body $\left(\sim 50{ }^{\circ} \mathrm{C}\right)$ for a well conditioned cesiated source. The electron density and the electron temperature can be reduced by a sufficient filter field (some $10 \mathrm{mT}$ ) as well as by a positive bias of the plasma grid with respect to the source body [3, 4, 9]. In this case, the electron temperature drops from about $10 \mathrm{eV}$ in the driver to less than the required $2 \mathrm{eV}$ near the plasma grid. The negative ion density in front of the plasma grid decreases from the grid towards the source center. Correspondingly, the electron density decreases more than 'normal' towards the plasma grid. In a well conditioned cesiated source, the number of negative ions in the region of a few $\mathrm{cm}$ in front of the plasma grid is comparable to the amount of electrons. At 2-3 cm distance, densities of $1-3 \times 10^{17} \mathrm{~m}^{-3}$ have been measured both for negative ions and electrons by cavity ringdown and optical emission spectroscopy [9].

Figure 2 shows the electrical and magnetic setup of the IPP RF source. The plasma grid is typically surrounded by a bias plate having the same potential as the source body. A diode prevents current reversal in the bias circuit; hence the plasma grid is floating, when the set bias voltage is too low [4]. The internal magnetic filter field can be weakened or strengthened by additional magnets outside the source.

The total path length of the negative ions from the place of birth at the plasma grid to the extraction aperture can be changed by the bias voltage, defining the starting energy of the ions by the potential between plasma grid and the bulk plasma, and by the grid geometry. There is an optimum of the ion energy: if the energy is too low, the ion cannot reach the next aperture, if the ion energy is too large, the ion path length is too large [8]. Using chamfered apertures makes the starting angle more favorable and the negative ion conversion area larger, as shown in experiments [3] and in calculations [8].

Figure 3 shows that the negative ion and electron transport can be decoupled. Here the extracted ion and electron current are shown during a D beam pulse at BATMAN when or when not a Langmuir probe was inserted into the plasma parallel the plasma grid. The probe was orientated perpendicular to the magnetic field lines. Powering the probe or not makes no difference in the electron suppression effect. There is no effect when the probe is oriented parallel to the field lines. Clearly, the effect on the extracted electron current is much more pronounced than for the negative ions. This means that the distance of $3 \mathrm{~cm}$ is far enough from the plasma grid not to influence the negative ion transport, hence the negative ion that are extracted are bent back within this region. On the other hand, the probe sits more or less in the maximum filter field (see figure 4), where the electron transport along the field lines is at maximum and the electron density is expected to have a maximum.

The negative ion and electron transport to the plasma grid can also be 'controlled' by changing the magnetic fields and the potentials in the source. A typical behavior of the RF source is that with increasing bias voltage at some value the electron current begins to decrease whereas the ion current is still high; the ion current starts then to decrease at a higher bias voltage [4]. For good performance, the bias voltage is set just above the floating potential of the grid so that some current can flow.

Another way to change the potential distribution is demonstrated in figure 5. Here the performance of the RF source is shown for $\mathrm{H}$ and $\mathrm{D}$ with and without bias plate. The internal filter field was optimized for D operation that means it was too large in order to achieve the maximum values for $\mathrm{H}$ as shown in figure 1 . In the case of $\mathrm{H}$, the same amount of ions can be extracted from the source in both cases. Without bias plate, however, the filter field has to be reduced, but this increases the amount of the coextracted electrons. The compensation of the current density decrease with a decrease of the filter field, however, is not possible in D. Due to the larger amount of co-extracted electrons, the filter field has to be larger than in $\mathrm{H}$; but only in the case with the bias plate the increase of the filter field does not decrease the ion current density.

The role of the bias plate and of the magnetic field is not quite well understood. For example, it is still not clear, which is the important parameter

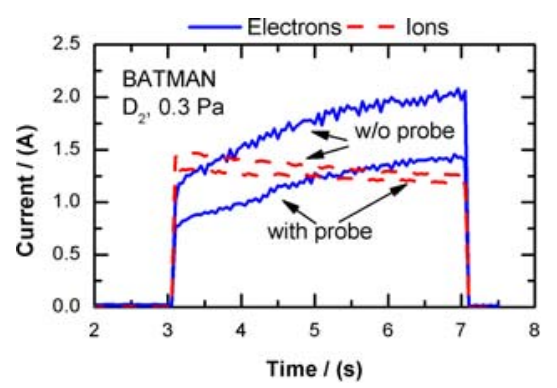

FIGURE 3. Electron and ion current at BATMAN with a probe inserted perpendicular to the magnetic filter field and parallel to the plasma grid in a distance of about $3 \mathrm{~cm}$ (see figure 1). 
for the electron suppression in the filter field, the $\int \mathrm{Bdl}$ or the filter field strength near the plasma grid, or the 3-dimensional structure of the field with edge effects. An indication of the latter is the large differences in the source performance even with small changes of the central filter field. Additionally, the electron deflection field from the extraction grid reaches also a few $\mathrm{cm}$ into the source and leads to further complexity. There is evidence from BATMAN that this field is necessary for a sufficient suppression of the co-extracted electrons [3].

A possible influence of the bias plate on the amount of the co-extracted electron is that the plasma is shifted away from the plasma grid and hence the electron density in front of the plasma grid is reduced. Measurements of the potential distribution with Langmuir probes are under way [10]. The effect of the bias plate on the ions cannot be separated from the electrons:

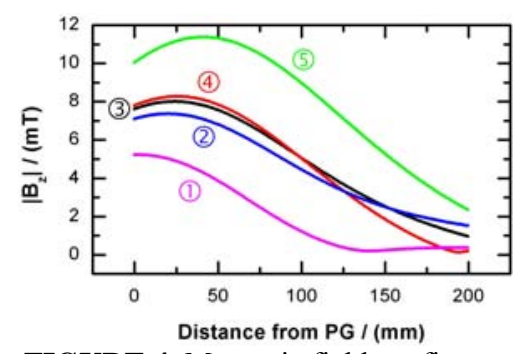

FIGURE 4. Magnetic field configurations in the center of the source. The field of the internal magnets (3) is weakened $(1,2)$ or strengthened $(4,5)$ by external magnets. if the amount of co-extracted electrons is too large, the RF power and the extraction voltage have to be limited in order to keep the power on the extraction grid reasonable low. This can be clearly seen in the case of $\mathrm{D}$ with the bias plate. On the other hand, there are also indications that the maximum achievable ion current density without bias plate is lower than with the bias plate for the same magnetic field configuration. This can be also explained by the larger electron density in front of the plasma grid without bias plate.

Figure 5 shows also, that the source efficiency in terms of extracted negative ion density is the same (or even larger) for D compared to $\mathrm{H}$. Hence, other means of electron suppression than by a filter field will give the possibility to reduce the (internal) filter field also for $\mathrm{D}$ and a current density of $300 \mathrm{~A} / \mathrm{m}^{2} \mathrm{D}^{-}$might be possible (see figure 1). A first example of alternative means of electron suppression in D was shown in figure 3; presently, corresponding experiments with an actively cooled rod in front of the plasma grid together with variations of the bias plate potential at MANITU are under way.

\section{CONCLUSION}

The Cs seeded IPP RF driven ion source is now the reference source for the ITER NBI system. The 'physical' ITER requirements with respect to current density and electron/ion ratio at the required pressure of $0.3 \mathrm{~Pa}$ have been obtained both for $\mathrm{H}$ and $\mathrm{D}$ for short pulses and a small extraction area. The understanding of the complex processes of negative ion production near the plasma grid on a cesiated surface and the subsequent extraction with sufficient electron suppression is evolving by measurements and modeling. This is important for the next steps of achieving the required ITER parameter also with an extended pulse length and source size.

\section{REFERENCES}

1. ITER EDA Documentation Series No. 24, Plant Description Document, Sec. 2.5.1. IAEA 2002

2. E. Speth et al. Plasma Science and Technology 6 (2004) 2135

3. E. Speth et al., Nuclear Fusion 46(6) (2006) S220

4. P. Franzen et al., Nuclear Fusion 47 (2007) 264

5. R. Hemsworth et al., Rev. Sci. Instr., accepted

6. P. Franzen et al., Fusion Engineering and Design 82 (2007) 407

7. W. Kraus et al., Rev. Sci. Instr., accepted

8. D. Wünderlich et al., Proc. of the $11^{\text {th }}$ PNNIB, Santa Fe, 2006.

9. U. Fantz et al., Rev. Sci. Instr., accepted

10. U. Fantz et al., Plasma Phys. and Contr. Fusion, accepted
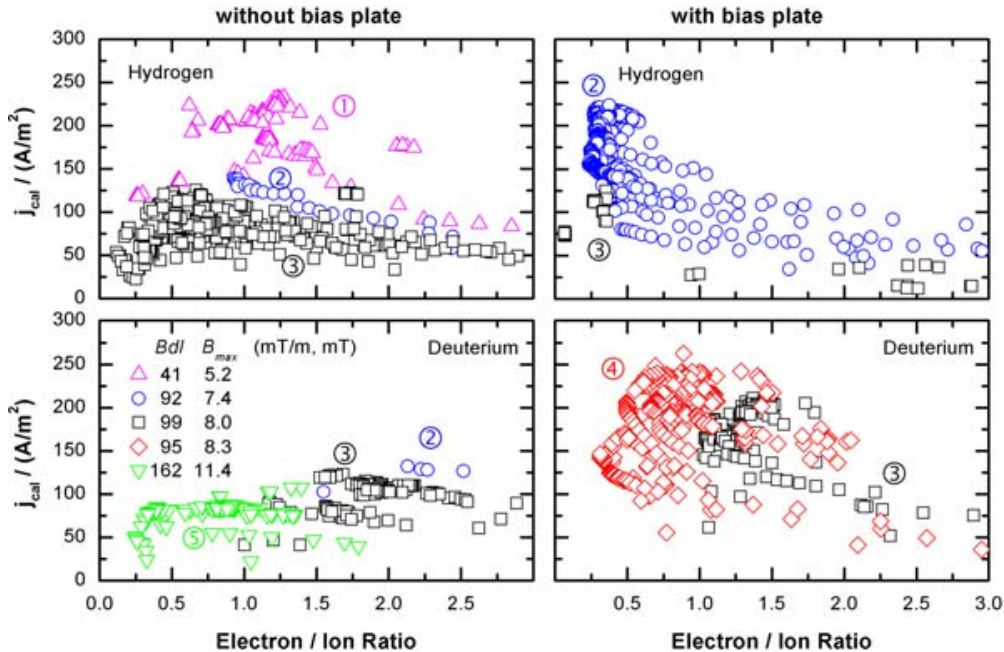

FIGURE 5. Dependence of the performance of the IPP RF source on the axial magnetic field configuration in the center of the source (see figure 4) with and without bias plate for $\mathrm{H}$ and $\mathrm{D}$. The internal filter field was the same both for $\mathrm{H}$ and $\mathrm{D}$ and stronger as for $\mathrm{H}$ in figure 1. 